(C) The Authors 2015. This is an Open Access article, distributed under the terms of the Creative Commons Attribution licence (http:// creativecommons.org/licenses/by/3.0/), which permits unrestricted re-use, distribution, and reproduction in any medium, provided the original work is properly cited.

\title{
Carbohydrate-rich breakfast attenuates glycaemic, insulinaemic and ghrelin response to ad libitum lunch relative to morning fasting in lean adults
}

\author{
Enhad A. Chowdhury ${ }^{1}$, Judith D. Richardson ${ }^{1}$, Kostas Tsintzas ${ }^{2}$, Dylan Thompson ${ }^{1}$ and James A. Betts ${ }^{1 *}$ \\ ${ }^{1}$ Department for Health, University of Bath, Bath BA2 7AY, UK \\ ${ }^{2}$ School of Life Sciences, Queen's Medical Centre, University of Nottingham, Nottingham, UK \\ (Submitted 29 January 2015 - Final revision received 27 March 2015 - Accepted 9 April 2015 - First published online 25 May 2015)
}

\begin{abstract}
Breakfast omission is associated with obesity and CVD/diabetes, but the acute effects of extended morning fasting upon subsequent energy intake and metabolic/hormonal responses have received less attention. In a randomised cross-over design, thirty-five lean men ( $n$ 14) and women $(n$ 21) extended their overnight fast or ingested a typical carbohydrate-rich breakfast in quantities relative to RMR (i.e. 1963 (SD 238) kJ), before an ad libitum lunch $3 \mathrm{~h}$ later. Blood samples were obtained hourly throughout the day until $3 \mathrm{~h}$ post-lunch, with subjective appetite measures assessed. Lunch intake was greater following extended fasting (640 (SD 1042) kJ, $P<0 \cdot 01$ ) but incompletely compensated for the omitted breakfast, with total intake lower than the breakfast trial (3887 (SD 1326) $v .5213$ (SD 1590) kJ, $P<0 \cdot 001$ ). Systemic concentrations of peptide tyrosine-tyrosine and leptin were greater during the afternoon following breakfast (both $P<0 \cdot 05$ ) but neither acylated/total ghrelin concentrations were suppressed by the ad libitum lunch in the breakfast trial, remaining greater than the morning fasting trial throughout the afternoon (all $P<0.05$ ). Insulin concentrations were greater during the afternoon in the morning fasting trial (all $P<0 \cdot 01$ ). There were no differences between trials in subjective appetite during the afternoon. In conclusion, morning fasting caused incomplete energy compensation at an ad libitum lunch. Breakfast increased some anorectic hormones during the afternoon but paradoxically abolished ghrelin suppression by the second meal. Extending morning fasting until lunch altered subsequent metabolic and hormonal responses but without greater appetite during the afternoon. The present study clarifies the impact of acute breakfast omission and adds novel insights into second-meal metabolism.
\end{abstract}

\section{Key words: Breakfast skipping: Appetite hormones: Insulin sensitivity: Second-meal effect: Energy intake}

Regular breakfast omission is associated with greater risk of obesity $^{(1,2)}$, prospective weight gain $^{(3)}$, type 2 diabetes ${ }^{(4,5)}$ and $\mathrm{CHD}^{(6)}$. Increased adiposity is due to chronic positive energy balance, so laboratory studies have understandably explored a possible role of daily breakfast in regulating energy intake (EI) and associated metabolic responses. However, the focus of previous research in adults has been to compare breakfasts of varied quantity or composition ${ }^{(7-14)}$. In contrast, the effects of a morning fasting condition $v$. breakfast consumption on EI and associated metabolic responses later in the same day has received less attention.

Laboratory studies in adults that have compared intake following breakfast omission have yielded equivocal results, with both reduced ${ }^{(15,16)}$ and similar ${ }^{(17)}$ overall EI observed. To the authors' knowledge, only two studies ${ }^{(15,17)}$ have measured hormonal and appetite responses to breakfast omission/ consumption followed by an ad libitum lunch. These studies therefore provide valuable information, but their designs included mid-morning preloads (i.e. a standardised feeding of $1050 \mathrm{~kJ}(250 \mathrm{kcal})$ and $1500 \mathrm{~kJ}(358 \mathrm{kcal})$ in all trials before lunch) such that the effects of unbroken overnight fasting prior to an ad libitum lunch were not a focus. However, there is evidence in adolescent girls that breakfast consumption can reduce hunger relative to morning fasting, with high protein breakfasts reducing daily ghrelin (an appetitestimulating hormone ${ }^{(18)}$ ), increasing peptide tyrosinetyrosine $^{(19)}$ (PYY; a hormone associated with satiety ${ }^{(20)}$ ) and reducing ad libitum lunch intake ${ }^{(21)}$, but in both cases without reducing total EI. In addition, the two preload studies ${ }^{(15,17)}$ described in adults have demonstrated that breakfast omission prior to a preload can affect subsequent metabolic responses along with hormonal outcomes such as PYY and glucagonlike peptide- $1^{(17)}$ (shown to augment insulin secretion to nutrients and reduce food intake ${ }^{(22)}$ ). Omission of breakfast therefore has the potential to affect EI as well as metabolic and hormonal responses to subsequent feeding.

The metabolic and hormonal responses to an ad libitum lunch when still in an overnight fasted state remain to be

Abbreviations: EI, energy intake; PYY, peptide tyrosine-tyrosine.

*Corresponding author: Dr J. A. Betts, fax +44 1225 383833, email j.betts@bath.ac.uk 
examined in adults. This question is of relevance both in terms of understanding the basic physiology influencing daily feeding patterns and in terms of practical relevance for the $19-28 \%$ of Western societies who frequently skip breakfast altogether $^{(23,24)}$. Accordingly, we examined acute EI, appetiteregulatory hormones and metabolic responses after extended morning fasting relative to a standardised high glycaemic index/carbohydrate breakfast. We hypothesised that the prescribed differences in EI during the morning would not be fully compensated for at lunch. Secondly, that morning fasting would result in greater appetite sensations consistent with increased orexigenic (ghrelin) and lower anorectic (e.g. PYY) hormone responses throughout the day.

\section{Methods \\ Participants}

The present study was part of a wider randomised controlled trial (the Bath Breakfast Project), other results from which have been published previously ${ }^{(25)}$, for which 301 individuals were invited for eligibility assessment, 231 were assessed for eligibility, 137 were invited to participate and thirty-eight agreed to do so (three dropped out prior to testing). Here we report data for thirty-five healthy, lean men $(n 14)$ and women ( $n$ 21) aged 22-56 years who completed this part of the project ${ }^{(26)}$. Within the present study cohort, there was a mix of frequent habitual breakfast consumers (classified as $>209 \mathrm{~kJ}$ ( $>50 \mathrm{kcal}$ ) intake within $2 \mathrm{~h}$ of waking on $\geq 4 \mathrm{~d}$ of the week; $n$ 27) and infrequent consumers ( $n$ 8). The sample size for this investigation was determined by estimates for the wider project described previously ${ }^{(26)}$. In brief, those estimates were based on the statistical power required to detect differences in free-living diet and physical activity between two independent groups ( $n$ 14). The total sample size for the current investigation $(n 35)$ is therefore more than adequate to confidently detect any meaningful within-subjects responses of the more tightly controlled laboratory-based measures assessed here using a repeated measures cross-over design. Participants were recruited via local advertisement from South West England and were initially assessed for eligibility based on a BMI of $18-25 \mathrm{~kg} / \mathrm{m}^{2}$ and later classified as lean based on dual-energy X-ray absorptiometry-derived fat mass indices of $\leq 7.5 \mathrm{~kg} / \mathrm{m}^{2}$ (men) and $\leq 11 \mathrm{~kg} / \mathrm{m}^{2}$ (women) ${ }^{(27)}$. Recruitment and laboratory visits spanned from 10 June 2010 to 16 May 2013. In accordance with the full eligibility criteria set out previously ${ }^{(26)}$, participants reported being weight stable ( $\pm 1 \mathrm{~kg}$ body mass within past 6 months), were free of metabolic disorders and adhered to a standard sleep-wake cycle (e.g. no shift workers). Participant characteristics are presented in Table 1.

\section{Ethical approval}

The present study was conducted according to the guidelines laid down in the Declaration of Helsinki and all procedures involving human subjects were approved by the National Health Service South West 3 Research Ethics Committee.
Table 1. Participant characteristics

(Mean values and standard deviations, $n$ 35)

\begin{tabular}{lcc}
\hline Characteristics & Mean & SD \\
\hline Age (years) & 36 & 11 \\
Body mass $(\mathrm{kg})$ & $67 \cdot 9$ & $9 \cdot 2$ \\
BMI $\left(\mathrm{kg} / \mathrm{m}^{2}\right)$ & $22 \cdot 7$ & $2 \cdot 5$ \\
Fat mass index* $\left(\mathrm{kg} / \mathrm{m}^{2}\right)$ & & \\
$\quad$ Female & $6 \cdot 7$ & $2 \cdot 0$ \\
$\quad$ Male & $4 \cdot 1$ & $1 \cdot 4$ \\
Habitual breakfast consumers† $(n)$ & 27 & \\
Female $(n)$ & 21 &
\end{tabular}

${ }^{*}$ Fat mass index calculated as dual-energy $\mathrm{X}$-ray absorptiometry-derived total fat mass divided by height squared.

$\dagger$ Habitual breakfast consumption defined as $>209 \mathrm{~kJ}$ (>50 kcal) intake within $2 \mathrm{~h}$ of waking on $\geq 4 \mathrm{~d} /$ week

Written informed consent was obtained from all participants. The present study is registered with Current Controlled Trials (http://www.isrctn.com) (ISRCTN31521726).

\section{Study design}

Each participant undertook a randomised, counterbalanced cross-over study design involving two laboratory-based feeding trials in the Human Physiology Laboratories at the University of Bath. Prior to their first day in the laboratory, participants recorded a 48-h weighed food and drink intake diary and repeated this prior to their second visit. In the $24 \mathrm{~h}$ prior to each laboratory visit, participants abstained from strenuous exercise, as well as caffeine and alcohol intake. These trials were separated by $3-28 \mathrm{~d}$ where participants resumed their habitual routine, with eumenorrheic women undergoing testing during the follicular phase of the menstrual cycle (3-10d after onset of menses) ${ }^{(28,29)}$. Dualenergy X-ray absorptiometry scans to assess the body composition of these participants occurred at a separate visit following the two laboratory visits described here.

\section{Protocol for laboratory visits}

On arrival at the laboratory at 08.00 hours $\pm 1 \mathrm{~h}$, following an overnight fast of $\geq 10 \mathrm{~h}$, participants voided and had body mass measured in light clothing (Seca 873; Vogel and Halke). RMR was assessed as described later, before a cannula was inserted into an antecubital vein, with a baseline sample of $15 \mathrm{ml}$ blood obtained. Participants were then provided with either a breakfast (to be consumed within $15 \mathrm{~min}$ ) or asked to rest for the same duration, with blood samples taken at $15 \mathrm{~min}, 30 \mathrm{~min}$ and an hour after completion of the breakfast period. Blood samples were then obtained hourly until $3 \mathrm{~h}$ post-breakfast, at which point an ad libitum lunch was provided. The lunch period was of 30-min duration with participants in isolation (i.e. all participants were tested entirely separately). Blood samples were obtained hourly from $1 \mathrm{~h}$ after completion of the lunch until $3 \mathrm{~h}$ after lunch. Participants also completed visual analogue scales relating to appetite throughout the day while remaining sedentary and completing quiet activities such as reading, watching 
television and typing. Participants remained in the laboratory throughout the duration of each testing visit.

\section{Breakfast}

The breakfast consisted of corn flakes (Kellogg's), $2 \%$ fat milk (Sainsbury's), toasted white bread (Braces), margarine (I can't believe it's not butter) and fresh orange juice (Sainsbury's) and was based on the breakfast provided by Chryssanthopoulos et $a l{ }^{(30)}$. Participants were given the choice of white sugar added to cornflakes, or seedless raspberry jam (Sainsbury's) on their toast, or an isoenergetic combination of both. The overall percentages of energy from macronutrients in the breakfast were $70 \%$ carbohydrate, $17 \%$ fat and $13 \%$ protein. This breakfast was selected because it is typical of the type of carbohydrate-rich foods commonly consumed at breakfast in the $\mathrm{UK}^{(24)}$ and also therefore provides an effective challenge to glycaemic control relative to the morning fasting trial. The breakfast was provided in quantities that contained $0.06 \mathrm{~g}$ carbohydrate per kcal of each individual participant's measured daily RMR, resulting in an EI of 1963 (SD 238) kJ (469 (SD 57) kcal). The size of this breakfast was based upon pilot work to determine an ecologically valid portion size, which also surpasses one of the most commonly accepted EI ranges of 'breakfast' as a minimum of $20 \%$ of daily $\mathrm{EI}^{(31)}$, whilst also standardising for individual differences in RMR as it directly reflects energy requirements during our resting trial and is a primary driver of $\mathrm{EI}^{(32)}$. Participants were first provided with cereal, then at 5-min intervals, toast and finally orange juice, with all of the breakfast consumed within 15 min to standardise any effects of eating rate upon appetite hormones ${ }^{(33)}$. During the morning fasting trial, participants sat quietly for a matched time period. No food or drink was allowed following consumption of the breakfast or completion of the matched rest period until the ad libitum lunch; therefore, in the breakfast trial, the overnight fasting duration was $\geq 11 \mathrm{~h}$ and in the morning fasting trial was $\geq 14.5 \mathrm{~h}$.

\section{Ad libitum lunch}

Three hours post-breakfast, participants were provided with an ad libitum lunch test meal consisting of $1 \mathrm{~kg}$ cooked (i.e. wet weight) penne pasta (Sainsbury's) including tomato sauce (Ragu); prepared at a ratio of 1:1 uncooked mass. Each pasta meal was prepared according to specified cooking times, with the energy density of the cooked pasta calculated (to account for any differences in water absorption) and the final masses of pasta and sauce combined noted, in order to calculate the energy density for the homogeneous mixture of pasta and sauce. The overall percentages of energy from macronutrients for the lunch were $79 \%$ carbohydrate, $14 \%$ fat and $7 \%$ protein. Consistent with the rationale for the carbohydrate-rich breakfast, this meal was selected on the basis that it would also challenge glycaemic control. Pasta was provided in a bowl which was replenished every $10 \mathrm{~min}$ to minimise visual feedback relating to consumed volume, and to prevent any tendency to finish the portion provided. Participants were left alone during the lunch with a recorded message played requesting participants eat until they had satisfied their hunger. During their first trial, participants were allowed ad libitum intake of plain water during lunch; this volume of water was subsequently replicated on their second visit.

\section{Expired gas analysis}

Expired air samples were collected in 200 litre Douglas bags (Hans Rudolph) via falconia tubing (Baxter, Woodhouse and Taylor), with concurrent measurement of inspired air composition $^{(34)}$. The obtained samples were passed through tubing containing anhydrous calcium sulphate (Drierite) to remove water content from the samples. Relative proportions of $\mathrm{O}_{2}$ and $\mathrm{CO}_{2}$ were measured in a known volume of the sample using paramagnetic and IR analysers, respectively (Servomex 1440). This analyser was calibrated on the morning of each testing day using two gases of known composition (British Oxygen Company). The volume of expired air was established using a dry gas meter (Harvard Apparatus), with the temperature of the expired gases measured using a CheckTemp1C (Hanna Instruments) during evacuation from the Douglas bags.

Samples for RMR were collected in accordance with guidelines for best practice by Compher et al. ${ }^{(35)}$. Rates of both $\mathrm{O}_{2}$ utilisation $\left(V_{\mathrm{O}_{2}}\right)$ and $\mathrm{CO}_{2}$ production $\left(V_{\mathrm{CO}_{2}}\right)$ were used to calculate energy expenditure ${ }^{(36)}$ corrected for urinary $\mathrm{N}$ excretion ${ }^{(37)}$ :

$$
\begin{aligned}
\text { Energy expenditure }= & \left(3.941 \times V_{\mathrm{O}_{2}}\right)+\left(1.106 \times V_{\mathrm{CO}_{2}}\right) \\
& +(2.17 \times \mathrm{N} \text { excretion }) .
\end{aligned}
$$

\section{Blood sampling}

The intravenous cannula was kept patent through regular flushing with $0.9 \% \mathrm{NaCl}$ infusion, with the first $5 \mathrm{ml}$ of each blood draw discarded. Whole blood was dispensed into tubes coated with EDTA for collection of plasma and immediately centrifuged. Serum was obtained by dispensing whole blood into a serum separation tube, which was left to stand at room temperature for $45 \mathrm{~min}$ before centrifugation. For analysis of acylated ghrelin, $1 \mathrm{ml}$ of whole blood was dispensed into a tube coated with EDTA, which had $50 \mu$ of a $p$-hydroxymercuribenzoic acid solution (prepared as a $100 \mathrm{~mm}$ concentrate solution in potassium phosphate buffer containing $1.2 \% 10 \mathrm{M}-\mathrm{NaOH})^{(38)}$. Samples were then centrifuged with $500 \mu \mathrm{l}$ of the supernatant transferred to an untreated blood tube containing $10 \mu \mathrm{l} 1 \mathrm{M}-\mathrm{HCl}$. Samples were centrifuged again and the supernatants removed. Centrifugation was at $3466 \mathrm{~g}$ for $10 \mathrm{~min}$ at $4{ }^{\circ} \mathrm{C}$ in all cases, with centrifuged samples immediately cooled using dry ice and then stored at $-80^{\circ} \mathrm{C}$ for subsequent analysis.

\section{Urine collection}

Participants' urine was collected in a vessel containing $5 \mathrm{ml}$ of $10 \%$ thymol isopropanol, which acted as a preservative. For a given measurement period, the collected urine was mixed 
thoroughly, with a $1 \mathrm{ml}$ aliquot obtained and kept at $-80^{\circ} \mathrm{C}$ prior to analysis. Analysis of urinary urea was conducted using a commercially available immunoassay as described later for plasma.

\section{Analysis of blood samples}

Total (intra-assay CV, $4 \cdot 0 \%$, inter-assay CV, 7.8\%) and acylated ghrelin (intra-assay CV, 4.2\%, inter-assay CV, 11.3\%) (Bertin Pharma), PYY (intra-assay CV, 4.3\%, inter-assay CV, 11.1\%) and total glucagon-like peptide-1 (intra-assay CV, $4.8 \%$, inter-assay $\mathrm{CV}, 27 \cdot 0 \%$ ) (Merck Millipore) assays were conducted using plasma, with leptin (intra-assay CV, 3.4\%, inter-assay CV, 6.4\%) (R\&D Systems, Inc.) and insulin (intraassay CV, $4.7 \%$, inter-assay CV, 12.5\%) (Mercodia AB) assays conducted using serum. All assays were done using commercially available ELISA, employed according to manufacturer's instructions, with all samples batches analysed at the conclusion of the study and samples from each participant included on the same plate. Analysis of blood samples for NEFA (intra-assay $\mathrm{CV},<5 \%$, inter-assay $\mathrm{CV},<5 \%$ ), glucose (intra-assay $\mathrm{CV},<5 \%$, inter-assay $\mathrm{CV},<6 \%$ ) and urea (intraassay $\mathrm{CV}, \quad<5 \%$, inter-assay $\mathrm{CV},<3 \%$ ) were conducted using plasma on a Daytona (Randox Laboratories) automated analyser according to manufacturer's instructions using commercially available immunoassays (Randox Laboratories).

\section{Appetite sensations}

Paper-based, 100-mm visual analogue scales were used to assess subjective appetite. These measurements were obtained immediately pre- and post-breakfast, immediately pre- and post-lunch and after a 3 -h postprandial period following lunch. Participants marked their response to questions assessing desire to eat, hunger, fullness and prospective consumption with anchor terms on each end of the scale (e.g. not at all hungry $v$. as hungry as I have ever felt). Higher scores were indicative of greater sensations. A composite appetite score $^{(39)}$ was calculated as follows:

$$
\begin{aligned}
\text { Composite appetite score }= & (\text { desire to eat }+ \text { hunger } \\
& +(100-\text { fullnesss }) \\
& + \text { prospective consumption }) / 4 .
\end{aligned}
$$

\section{Statistical analysis}

For single comparisons of two means (e.g. EI at lunch), the distribution of all data was verified as normal using a ShapiroWilk test and paired $t$ tests were thus employed throughout. For comparison of time series variables that were measured over the course of the day in each condition (e.g. appetite hormones), repeated measures ANOVA (breakfast/fasting $\times$ time point) were employed with Greenhouse-Geisser corrections applied to intra-individual contrasts for $\varepsilon<0.75$, and the Huynh-Feldt correction applied for less severe asphericity ${ }^{(40)}$. Significant interactions were explored with multiple $t$ tests to locate variance between trials at level time points, with a Holm-Bonferroni stepwise adjustment ${ }^{(41)}$. Statistical significance was accepted at $P \leq 0 \cdot 05$. Data are presented in text as means and standard deviations, figures display mean with normalised CI. These CI represent the comparison between the two trials, removing the inter-individual variation due to the fully paired nature of the experimental design ${ }^{(42)}$. All statistical analyses were conducted using IBM SPSS statistics version 22 (IBM)

\section{Results}

\section{Energy intake}

During the breakfast trial, participants consumed a prescribed breakfast of 1963 (SD 238) kJ (469 (SD 57) kcal) (i.e. variance proportionate to inter-individual differences in RMR). During the ad libitum lunch, participants consumed 3247 (SD 1460) kJ (776 (sD 349) kcal) following breakfast but significantly more in the morning fasting trial (3887 (SD 1326) kJ (929 (sD 317) kcal); $P<0.01$, Fig. 1). However, when the absolute EI during the breakfast trial was calculated (breakfast + lunch), this was significantly more than during the morning fasting trial (5213 (SD 1590) v. 3887 (SD 1326) kJ (1246 (SD 380) v. 929 (sD 317) kcal); $P<0 \cdot 001$ ). When comparing the EI at lunch in the two conditions, the additional EI of 640 (SD 1042) kJ (153 (SD 249) kcal) during the morning fasting trial accounted for approximately $33 \%$ of the prescribed breakfast (i.e. additional intake at lunch during the fasting trial was insufficient to compensate for the breakfast provided).

\section{Glucose}

There were main effects of time, trial and a trial $\times$ time interaction for plasma glucose concentrations (all $P<0.01$ ). Glucose concentrations were significantly greater than fasting following breakfast consumption until $2 \mathrm{~h}$ post-breakfast (all $P<0 \cdot 03$, Fig. 2(a)). By $3 \mathrm{~h}$ post-breakfast, there was no

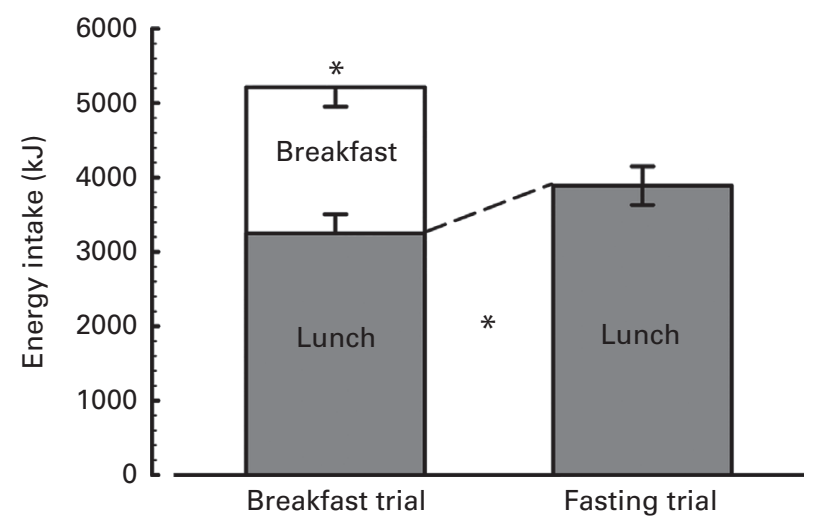

Fig. 1. Energy intake during trials. In the morning fasting trial, an asymmetric normalised $\mathrm{Cl}$ is plotted, the negative portion of which reflects the comparison between lunches and the positive portion reflects the comparison against total intake (i.e. lunch plus breakfast). An asterisk above a bar represents the comparison between the sum of the components of the bar, an asterisk between the bars represents the comparison between the specific component $(P<0.01)$. $n 34$, as one individual felt nauseous prior to lunch provision on one visit. 
difference in plasma glucose concentrations between trials $(P=0 \cdot 3)$. Glucose concentrations were significantly greater $1 \mathrm{~h}$ post-lunch in the morning fasting trial $(P<0 \cdot 01)$. There was a strong tendency for greater concentrations in the morning fasting trial at $2 \mathrm{~h}$ post-lunch (breakfast trial, 5.99 (SD 0.75$) \mathrm{mm} v$. fasting trial, 6.49 (SD 1.02$) \mathrm{mm} ; P=0.06$ ) but no difference between trials $3 \mathrm{~h}$ after lunch $(P=0 \cdot 6)$.

\section{Insulin}

There were main effects of time, trial and a trial $\times$ time interaction for serum insulin concentrations (all $P<0 \cdot 01$ ). Following breakfast consumption, insulin concentrations were significantly greater during the morning than during the fasting trial (all $P<0.01$, Fig. 2(b)). Following lunch, insulin concentrations were significantly greater in the morning fasting trial than in the breakfast trial (all $P<0 \cdot 01$ ).

\section{NEFA}

There were main effects of time, trial and a trial $\times$ time interaction for plasma NEFA concentrations (all $P<0 \cdot 01$ ). NEFA concentrations were suppressed following breakfast consumption throughout the day but remained elevated in the morning fasting trial prior to lunch (all $P<0.01$, Fig. 2(c)). Following lunch, NEFA concentrations were strongly suppressed by feeding in the morning fasting trial such that there was no difference between the trials (all $P>0.05$ ).

\section{Acylated and total ghrelin}

There were main effects of time and a trial $\times$ time interaction for plasma acylated ghrelin (both $P<0 \cdot 01$ ). Breakfast consumption suppressed acylated ghrelin concentrations such that these were significantly lower 1 and $2 \mathrm{~h}$ post-breakfast relative to the morning fasting trial (both $P<0 \cdot 01$, Fig. 3(a)), but there was no difference between trials $3 \mathrm{~h}$ post-breakfast. Acylated ghrelin concentrations were significantly greater than in the morning fasting trial throughout the afternoon in the breakfast trial (all $P<0 \cdot 01$ ).

There were main effects of time, trial and a trial $\times$ time interaction for plasma total ghrelin concentrations (all $P<0 \cdot 01$ ). Following breakfast consumption, total ghrelin concentrations were suppressed, resulting in significantly lower concentrations than in the morning fasting trial prior to lunch (all $P<0 \cdot 01$, Fig. 3(a)). Following lunch, there was greater suppression of total ghrelin in the morning fasting trial, such that the concentrations were significantly lower than in the breakfast trial throughout the afternoon (all $P \leq 0 \cdot 05$ ).

\section{Peptide tyrosine-tyrosine}

There were main effects of time, trial and a trial $\times$ time interaction for plasma PYY concentrations (all $P<0 \cdot 01$ ). Greater PYY concentrations throughout the day following breakfast consumption were apparent $(P<0 \cdot 05$, Fig. 3(b)).
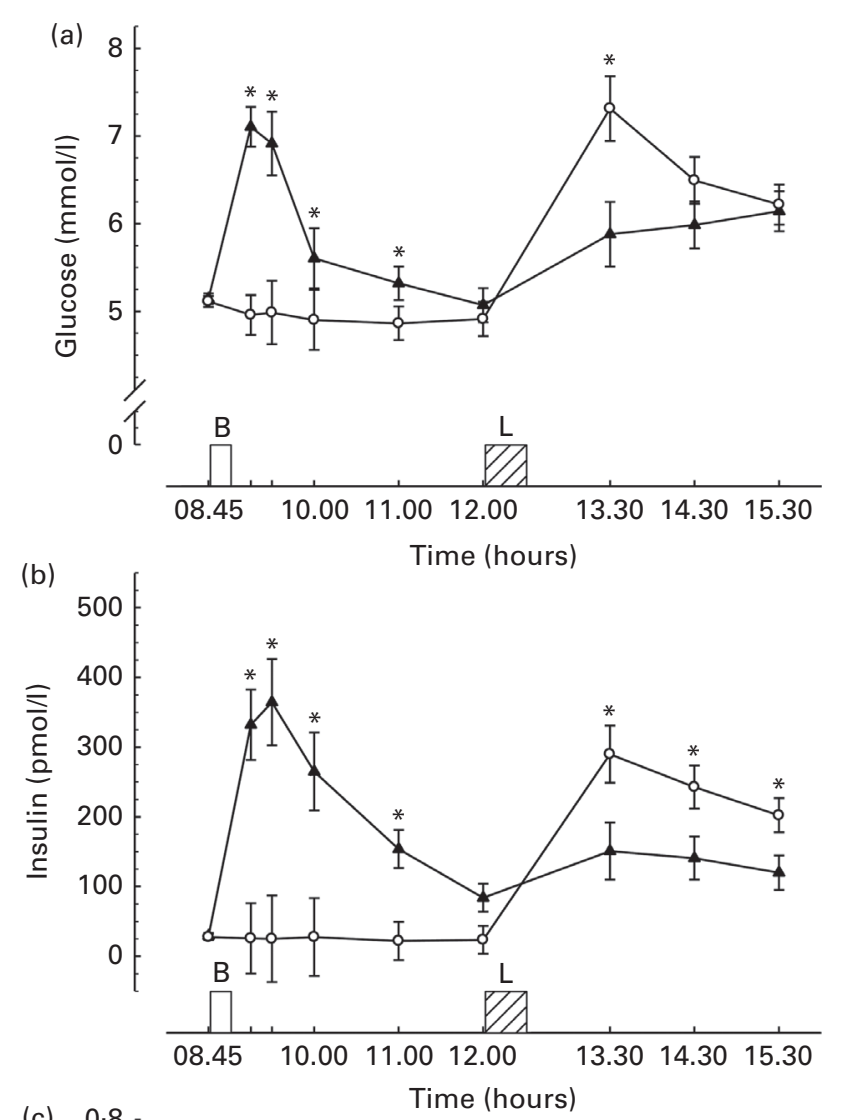

Fig. 2. Metabolic responses during trials. (a) Plasma glucose (n 32), (b) serum insulin ( $n$ 32), (c) plasma NEFA ( $n$ 31), where missing data are due to insufficient blood for analysis. Values are means with their normalised $\mathrm{Cl}$ represented by vertical bars. ${ }^{*}$ Mean value was significantly different from the corresponding time point in other trial $(P<0.03)$. B, breakfast period, in which participants ate a prescribed breakfast during the breakfast trial and rested during the morning fasting trial. L, ad libitum pasta lunch.

$-\boldsymbol{\Delta}-$, Breakfast; -O-, fasting.

\section{Glucagon-like peptide-1}

There was a tendency for a main effect of time $(F=2 \cdot 27$, $P=0 \cdot 08)$, but no effect of trial $(F=2 \cdot 89, P=0 \cdot 1)$ for plasma glucagon-like peptide- 1 concentrations. There was a trial $\times$ time interaction $(F=3.09, P=0.03)$ for glucagon-like peptide-1, with greater concentrations observed at $2 \mathrm{~h}$ after breakfast consumption when compared with the morning fasting trial but no differences between trials after lunch (Fig. 3(c)). 

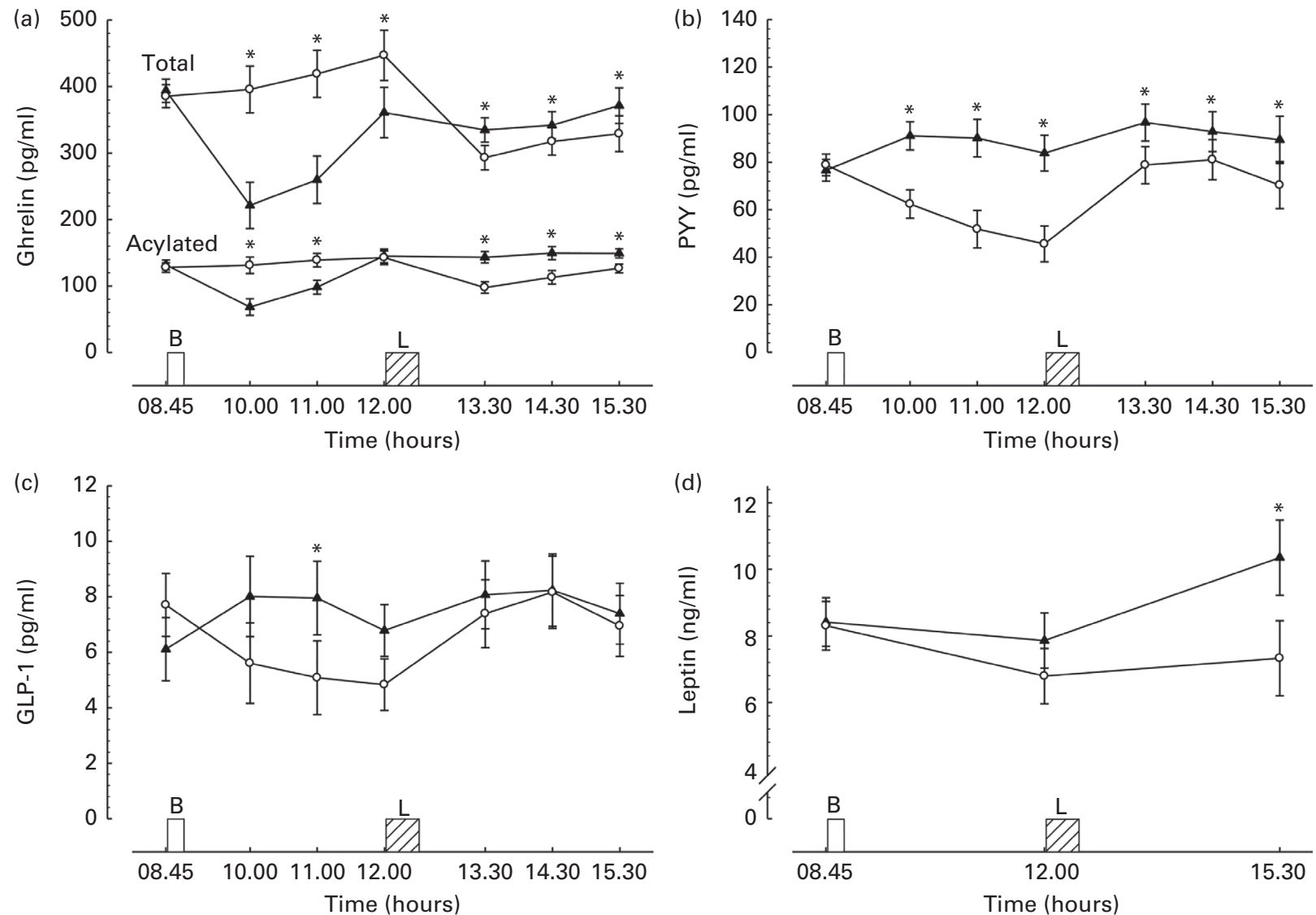

Fig. 3. Hormonal responses during trials. (a) Plasma acylated and total ghrelin ( $n$ 32), (b) plasma peptide tyrosine-tyrosine (PYY, $n$ 32), (c) plasma glucagon-like peptide-1 (GLP-1, $n$ 32), (d) serum leptin ( $n$ 32), where missing data are due to insufficient blood for analysis. Values are means with their normalised $\mathrm{Cl}$ represented by vertical bars. * Mean value was significantly different from the corresponding time point in other trial $(P<0.05)$. B, breakfast period, in which participants ate a prescribed breakfast during the breakfast trial and rested during the morning fasting trial. L, ad libitum pasta lunch. $-\mathbf{\Lambda}-$, Breakfast; $-\bigcirc-$, fasting.

\section{Leptin}

Serum leptin concentrations in both trials are displayed in Fig. 3(d). There were main effects of time, trial and a trial $\times$ time interaction for leptin (all $P<0.02$ ). In the breakfast trial, leptin concentrations were reduced from baseline at $3 \mathrm{~h}$ postbreakfast, but this reduction was greater in the morning fasting trial, such that there was a trend for lower leptin concentrations (breakfast trial, $7.86(\mathrm{sD} 7.28) \mathrm{ng} / \mathrm{ml} v$. fasting trial, 6.79 (SD 6.63) $\mathrm{ng} / \mathrm{ml} ; P=0 \cdot 07)$. Three hours post-lunch, leptin concentrations had increased in the breakfast trial to a greater extent than in the morning fasting trial, resulting in significantly greater concentrations of leptin in the breakfast trial $(P<0 \cdot 01)$.

\section{Subjective appetite ratings}

The composite appetite score combining the hedonics obtained is displayed in Fig. 4. Desire to eat, hunger and prospective consumption all followed very similar patterns throughout the day. Immediately after breakfast consumption, there was a reduction in all three measures such that there were significant differences between all three measures compared with the morning fasting trial (all $P<0 \cdot 01$, data not shown). Immediately prior to lunch, these appetite sensations remained greater in the morning fasting than in the breakfast trial (all $P<0 \cdot 01$ ). Following the consumption of the ad libitum lunch, all three measures decreased to a nadir with no significant difference between trials (all $P>0 \cdot 4$ ). Three hours after completion of lunch, there was no difference between trials for any of the measures (all $P \geq 0 \cdot 1$ ). The sensations of fullness followed a similar, but opposite, pattern throughout the day, with fullness significantly greater after breakfast and prior to lunch in the breakfast trial (both $P<0.01$ ) but not different between trials at any other time point (all $P>0 \cdot 2$ ).

\section{Discussion}

The present study investigated EI along with hormonal, metabolic and subjective appetite responses to an ad libitum lunch in an overnight fasted state in contrast with following a standardised breakfast in lean individuals. EI at the ad libitum lunch was greater following morning fasting than breakfast. However, in accordance with our hypothesis, greater lunch intake was insufficient to compensate for energy from breakfast, resulting in lower net intake in the morning fasting trial Following lunch, the response of hormones associated with satiety (PYY, leptin) was greater in the breakfast trial but, paradoxically, acylated/total ghrelin were not suppressed after lunch in the breakfast trial. Suppression of ghrelin would be 


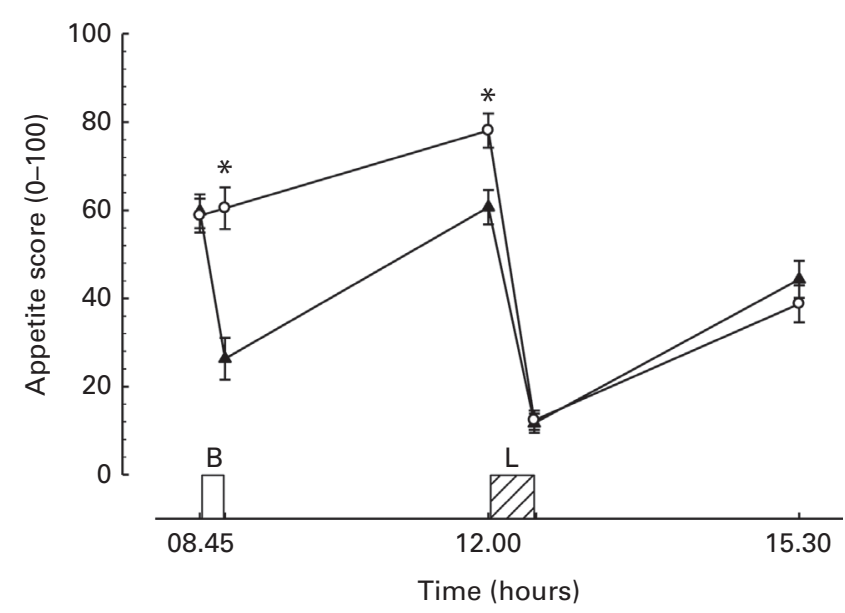

Fig. 4. Appetite score during trials. B, breakfast period, in which participants ate a prescribed breakfast during the breakfast trial and rested during the morning fasting trial. L, ad libitum pasta lunch. $n 34$, as one individual was not provided with hedonic scales on one of their trials. Values are means with their normalised $\mathrm{Cl}$ represented by vertical bars. * Mean value was significantly different from the corresponding time point in other trial $(P<0.01)$.

$-\boldsymbol{\Delta}-$, Breakfast; -O-, fasting.

expected due to the substantial energy/carbohydrate intake at lunch. In contrast to our hypothesis, subjective appetite $3 \mathrm{~h}$ after lunch was not different between trials; indicating that morning fasting may not cause greater appetite than breakfast in the afternoon despite incomplete compensation at lunch and lower concentrations of satiety-inducing hormones. This may be due to abolished ghrelin suppression after lunch following breakfast.

PYY has been shown to reduce food intake(20) with increased caloric load increasing concentrations ${ }^{(38)}$. In the present study, PYY increased in response to breakfast, remaining higher than the morning fasting trial throughout the day, despite greater intake at lunch following morning fasting. This is consistent with the accumulated difference in intake, and is supported by a report of similar PYY concentrations between conditions after an ad libitum lunch at which participants ate sufficient to compensate energetically for an omitted breakfast ${ }^{(17)}$. In combination, this suggests PYY is more a reflection of nutritional status over the entire day rather than in response to the most recent feeding, consistent with PYY peaking $1-2 \mathrm{~h}$ after feeding, followed by an elevation lasting several hours ${ }^{(43)}$.

Leptin concentrations were also greater $3 \mathrm{~h}$ after lunch in the breakfast trial. This finding is in accord with the slow response of leptin to feeding, such that increased concentrations relative to the morning fasting trial approximately $6 \mathrm{~h}$ after breakfast are likely a product of postprandial glucose and insulin responses to breakfast and lunch ${ }^{(44)}$. It is possible that the diurnal phase of leptin may have shifted in the morning fasting trial such that the usual increase later in the day was delayed. Indeed, postponing food intake from 07.00 to 13.30 hours has been shown to shift the rise in leptin later in the day ${ }^{(45)}$

The greater PYY and leptin during the afternoon in the breakfast trial would be expected to contribute to greater satiety at the end of the trial, although there was no difference in subjective appetite between the trials $3 \mathrm{~h}$ after lunch. This may be due to the complete lack of ghrelin suppression following lunch in the breakfast trial. This is a particularly unusual result that, to our knowledge, has not been reported previously in lean individuals. As increasing energy content of liquid breakfasts with carbohydrate causes greater reductions in ghrelin ${ }^{(46)}$, it is particularly puzzling that the ad libitum lunch had no impact on ghrelin as the lunch during the breakfast trial was 3247 (SD 1460) kJ (776 (SD 349) kcal) and rich in carbohydrate. Ghrelin has been associated with meal initiation $^{(18)}$ and as such it would be unexpected for this hormone to remain elevated after the second meal of the day. Foster-Schubert et al. ${ }^{(47)}$ have shown that an $80 \%$ carbohydrate breakfast drink (providing 20\% of daily energy requirements) suppressed ghrelin initially, but concentrations subsequently rebounded above fasting concentrations after $3 \mathrm{~h}$, remaining elevated for the majority of the next $3 \mathrm{~h}$. An obvious distinction presently is that $3 \mathrm{~h}$ after initial feeding, the participants received another carbohydrate-rich meal. This indicates that in the breakfast trial the ghrelin response to the second meal was abolished, with ghrelin following the established time course of a similar carbohydrate load without a second feeding occasion.

There have been reports that insulin may play an important role in ghrelin suppression ${ }^{(46,48-50)}$, although this view is not universal $^{(51,52)}$, with some authors suggesting that food intake specifically suppresses ghrelin but not the administration of insulin ${ }^{(51)}$. It is interesting to note that when consuming breakfast, insulin responses were significantly diminished after lunch relative to the morning fasting trial. It is conceivable the substantial reduction in insulin concentrations during the afternoon may have contributed to the absence of ghrelin suppression after lunch in the breakfast trial. However, this lack of suppression needs confirmation, and it remains to be seen whether elevated ghrelin following a lunchtime meal as observed translates to greater intake throughout the rest of the day. It has been suggested in a time-blinded study that ghrelin needs to reach a 'threshold' (reported as $93 \%$ of fasting concentrations) prior to meal requests ${ }^{(53)}$. As ghrelin in the morning fasting trial had returned to baseline concentrations $3 \mathrm{~h}$ after lunch (the point at which appetite was assessed in the afternoon), this may explain why there was no difference in appetite detected despite greater concentrations of ghrelin in the breakfast trial. In addition, as previously discussed, although ghrelin was greater throughout the afternoon, anorectic hormones were also elevated in the breakfast trial. Finally, it may be that ghrelin concentrations following repeated meals may not be as relevant in signalling hunger as prior to the first/ second meal of the day.

Whilst reduced insulin and glucose concentrations after lunch may be partly explained by the slight reduction 640 (SD 1042) kJ (153 (SD 249) kcal) in lunch intake following breakfast, this is also potentially representative of a second meal effect on glucose ${ }^{(54)}$, with an associated reduction of insulin concentration and potentiation of its effects ${ }^{(55)}$. Evidence that reduced insulin concentrations after lunch were due to the prior meal and not just reduced intake is 
demonstrated in the eleven individuals who ate similar lunches in both trials (i.e. more in the breakfast trial or intake within $335 \mathrm{~kJ}$ ( $80 \mathrm{kcal}$ ) in both trials). Ten of these individuals had reduced insulin concentrations $60 \mathrm{~min}$ after lunch during the breakfast trial, with concentrations 68 (SD 25) \% of the morning fasting trial, providing evidence of acutely reduced insulin sensitivity following extended fasting.

Previous studies both support ${ }^{(17)}$ and oppose the possibility that breakfast omission is compensated for at lunch ${ }^{(15)}$, with both studies including a mid-morning preload (i.e. a fixed feeding occasion $90 \mathrm{~min}$ before lunch). When this 'snack' feeding prior to lunch is removed and the fast remains unbroken until the ad libitum lunch, Levitsky \& Pacanowski ${ }^{(16)}$ report that intake at lunch was either unaffected, or was reduced by $728 \mathrm{~kJ}$ (approximately $174 \mathrm{kcal}$ ), following prescribed $1464 \mathrm{~kJ}(350 \mathrm{kcal})$ breakfasts or a self-selected $2611 \mathrm{~kJ}$ (approximately $624 \mathrm{kcal}$ ) buffet breakfast, respectively. Lunch intake in the present study was only reduced to a similar degree as the larger breakfast in the aforementioned study. Therefore, it appears that any persistent satiating effect of a typical breakfast at lunch is only brought about by breakfast intake of a magnitude that cannot reasonably be matched solely by reduced intake at lunch.

A limitation of the present study is that without intake data for the remainder of the day after lunch, it is not possible to establish whether further energetic compensation would occur within the 24-h period. However, laboratory work measuring intake following lunch through the afternoon/ evening (including snacking opportunities) has not identified greater consumption at any feeding occasion following lunch after morning fasting ${ }^{(16)}$. Limited daily compensation has also been observed when fasting through the morning during free living ${ }^{(25,56)}$. Another potential limitation is that, whilst each individual participant repeated dietary intake for $48 \mathrm{~h}$ prior to each laboratory visit, this pre-trial diet was not standardised between participants. While any variance between different individuals would not confound the overall interpretation between trials within this repeated measures design, it could contribute to greater variability in interindividual responsiveness between treatments, given that an extreme shift in macronutrient composition of an evening meal (i.e. $16 v$. $62 \%$ energy as fat) can affect next-day metabolic outcomes ${ }^{(57)}$. However, an additional consideration is that a standardised evening meal would not represent habitual intake for each individual. It should also be noted that appetite sensations were obtained relatively infrequently and this may have reduced our ability to detect differences between trials during the afternoon.

Future laboratory studies should include further feeding opportunities after lunch while extending metabolic and hormonal measures through the evening to elucidate the role of these physiological responses in influencing feeding throughout the duration of the day after morning fasting. As the first study to report the unexpected response of ghrelin to repeated carbohydrate-rich/high glycaemic index feedings, it is important for further research to replicate these findings and also examine breakfasts of different macronutrient composition and glycaemic index. In addition, due to the suggested appetite suppressive effects of higher protein breakfasts in adolescents ${ }^{(19,21)}$ and during the morning in adults $^{(58)}$, the effects of sequential meals on metabolic and hormonal responses following high protein breakfasts should be further examined in adults.

In summary, while morning fasting was incompletely compensated for at an ad libitum lunch, prior carbohydraterich breakfast consumption increased concentrations of some satiety hormones after lunch but abolished suppression of the orexigenic hormone ghrelin. This novel finding may be mediated through reduced insulin response to a second meal and results in similar appetite during the afternoon independent of morning feeding pattern.

\section{Acknowledgements}

We would like to acknowledge assistance provided by Matthew Jeans with elements of data collection.

The present study was supported by a grant from the Biotechnology and Biological Sciences Research Council (BB/H008322/1). E. A. C. received a University Research Scholarship from the University of Bath. The Biotechnology and Biological Sciences Research Council had no role in the design, analysis or writing of this article.

All data created during this research are openly available from the University of Bath data archive at http://dx.doi.org/ 10.15125/BATH-00092

The authors' contributions are as follows: J. A. B., D. T., J. D. R., E. A. C. and K. T. designed research; J. D. R., E. A. C., J. A. B. and D. T. conducted research; E. A. C., J. A. B. and J. D. R. analysed data and performed statistical analysis; E. A. C. and J. A. B. wrote the paper; E. A. C. had primary responsibility for final content. All authors read and approved the final manuscript.

The authors declare that there are no conflicts of interest.

\section{References}

1. Ma Y, Bertone ER, Stanek EJ, et al. (2003) Association between eating patterns and obesity in a free-living US adult population. Am J Epidemiol 158, 85-92.

2. Horikawa C, Kodama S, Yachi Y, et al. (2011) Skipping breakfast and prevalence of overweight and obesity in Asian and Pacific regions: a meta-analysis. Prev Med 53, 260-267.

3. Purslow LR, Sandhu MS, Forouhi N, et al. (2008) Energy intake at breakfast and weight change: prospective study of 6,764 middle-aged men and women. Am J Epidemiol 167, 188-192.

4. Mekary RA, Giovannucci E, Cahill L, et al. (2013) Eating patterns and type 2 diabetes risk in older women: breakfast consumption and eating frequency. Am J Clin Nutr 98, 436-443.

5. Mekary RA, Giovannucci E, Willett WC, et al. (2012) Eating patterns and type 2 diabetes risk in men: breakfast omission, eating frequency, and snacking. Am J Clin Nutr 95, 1182-1189.

6. Cahill LE, Chiuve SE, Mekary RA, et al. (2013) Prospective study of breakfast eating and incident coronary heart disease 
in a cohort of male US health professionals. Circulation $\mathbf{1 2 8}$, $337-343$.

7. Clegg M \& Shafat A (2010) Energy and macronutrient composition of breakfast affect gastric emptying of lunch and subsequent food intake, satiety and satiation. Appetite 54, $517-523$

8. Hamedani A, Akhavan T, Samra RA, et al. (2009) Reduced energy intake at breakfast is not compensated for at lunch if a high-insoluble-fiber cereal replaces a low-fiber cereal. Am J Clin Nutr 89, 1343-1349.

9. Kim H, Stote KS, Behall KM, et al. (2009) Glucose and insulin responses to whole grain breakfasts varying in soluble fiber, $\beta$-glucan: a dose response study in obese women with increased risk for insulin resistance. Eur J Nutr 48, 170-175.

10. Levine AS, Tallman JR, Grace MK, et al. (1989) Effect of breakfast cereals on short-term food intake. Am J Clin Nutr 50, 1303-1307.

11. Liljeberg HG, Akerberg AK \& Bjorck IM (1999) Effect of the glycemic index and content of indigestible carbohydrates of cereal-based breakfast meals on glucose tolerance at lunch in healthy subjects. Am J Clin Nutr 69, 647-655.

12. Martin A, Normand S, Sothier M, et al. (2000) Is advice for breakfast consumption justified? Results from a short-term dietary and metabolic experiment in young healthy men. Br J Nutr 84, 337-344.

13. Rosen LA, Ostman EM \& Bjorck IM (2011) Effects of cereal breakfasts on postprandial glucose, appetite regulation and voluntary energy intake at a subsequent standardized lunch; focusing on rye products. Nutr J 10,7.

14. Nilsson AC, Ostman EM, Granfeldt Y, et al. (2008) Effect of cereal test breakfasts differing in glycemic index and content of indigestible carbohydrates on daylong glucose tolerance in healthy subjects. Am J Clin Nutr 87, 645-654.

15. Gonzalez JT, Veasey RC, Rumbold PL, et al. (2013) Breakfast and exercise contingently affect postprandial metabolism and energy balance in physically active males. Br J Nutr 110, 721-732

16. Levitsky DA \& Pacanowski CR (2013) Effect of skipping breakfast on subsequent energy intake. Physiol Behav 119 , 9-16.

17. Astbury NM, Taylor MA \& Macdonald IA (2011) Breakfast consumption affects appetite, energy intake, and the metabolic and endocrine responses to foods consumed later in the day in male habitual breakfast eaters. J Nutr 141, 1381-1389.

18. Cummings DE, Purnell JQ, Frayo RS, et al. (2001) A preprandial rise in plasma ghrelin levels suggests a role in meal initiation in humans. Diabetes 50, 1714-1719.

19. Leidy HJ, Ortinau LC, Douglas SM, et al. (2013) Beneficial effects of a higher-protein breakfast on the appetitive, hormonal, and neural signals controlling energy intake regulation in overweight/obese, "breakfast-skipping," late-adolescent girls. Am J Clin Nutr 97, 677-688.

20. Batterham RL, Cowley MA, Small CJ, et al. (2002) Gut hormone PYY(3-36) physiologically inhibits food intake. Nature 418, 650-654.

21. Leidy HJ \& Racki EM (2010) The addition of a protein-rich breakfast and its effects on acute appetite control and food intake in 'breakfast-skipping' adolescents. Int J Obes (Lond) 34, 1125-1133.

22. Holst JJ (2013) Incretin hormones and the satiation signal. Int J Obes (Lond) 37, 1161-1168.

23. Kant AK \& Graubard BI (2014) 40-Year trends in meal and snack eating behaviors of American adults. I Acad Nutr Diet 115, 50-63.
24. Reeves S, Halsey LG, McMeel Y, et al. (2013) Breakfast habits, beliefs and measures of health and wellbeing in a nationally representative UK sample. Appetite 60, 51-57.

25. Betts JA, Richardson JD, Chowdhury EA, et al. (2014) The causal role of breakfast in energy balance and health: a randomized controlled trial in lean adults. Am J Clin Nutr 100, 539-547.

26. Betts JA, Thompson D, Richardson JD, et al. (2011) Bath Breakfast Project (BBP) - examining the role of extended daily fasting in human energy balance and associated health outcomes: study protocol for a randomised controlled trial [ISRCTN31521726]. Trials 12, 172.

27. Kelly TL, Wilson KE \& Heymsfield SB (2009) Dual energy X-ray absorptiometry body composition reference values from NHANES. PLOS ONE 4, e7038.

28. Buffenstein R, Poppitt SD, McDevitt RM, et al. (1995) Food intake and the menstrual cycle: a retrospective analysis, with implications for appetite research. Physiol Behav 58, 1067-1077.

29. Lissner L, Stevens J, Levitsky DA, et al. (1988) Variation in energy intake during the menstrual cycle: implications for food-intake research. Am J Clin Nutr 48, 956-962.

30. Chryssanthopoulos C, Williams C, Nowitz A, et al. (2004) Skeletal muscle glycogen concentration and metabolic responses following a high glycaemic carbohydrate breakfast. J Sports Sci 22, 1065-1071.

31. Timlin MT \& Pereira MA (2007) Breakfast frequency and quality in the etiology of adult obesity and chronic diseases. Nutr Rev 65, 268-281.

32. Blundell JE, Caudwell P, Gibbons C, et al. (2012) Role of resting metabolic rate and energy expenditure in hunger and appetite control: a new formulation. Dis Model Mech 5, 608-613.

33. Kokkinos A, le Roux CW, Alexiadou K, et al. (2010) Eating slowly increases the postprandial response of the anorexigenic gut hormones, peptide $\mathrm{YY}$ and glucagon-like peptide-1. J Clin Endocrinol Metab 95, 333-337.

34. Betts JA \& Thompson D (2012) Thinking outside the bag (not necessarily outside the lab). Med Sci Sports Exerc 44 , 2040; author reply 2041.

35. Compher C, Frankenfield D, Keim N, et al. (2006) Best practice methods to apply to measurement of resting metabolic rate in adults: a systematic review. J Am Diet Assoc 106, $881-903$.

36. Weir JB (1949) New methods for calculating metabolic rate with special reference to protein metabolism. J Physiol 109, 1-9.

37. Jequier E, Acheson K \& Schutz Y (1987) Assessment of energy expenditure and fuel utilization in man. Ann Rev Nutr 7, 187-208.

38. Chandarana K, Drew ME, Emmanuel J, et al. (2009) Subject standardization, acclimatization, and sample processing affect gut hormone levels and appetite in humans. Gastroenterology 136, 2115-2126.

39. Anderson GH, Catherine NL, Woodend DM, et al. (2002) Inverse association between the effect of carbohydrates on blood glucose and subsequent short-term food intake in young men. Am J Clin Nutr 76, 1023-1030.

40. Atkinson G (2002) Analysis of repeated measurements in physical therapy research: multiple comparisons amongst level means and multi-factorial designs. Phys Ther Sport 3, 191-203.

41. Ludbrook J (1998) Multiple comparison procedures updated. Clin Exp Pharmacol Physiol 25, 1032-1037.

42. Loftus GR \& Masson ME (1994) Using confidence intervals in within-subject designs. Psychon Bull Rev 1, 476-490. 
43. Adrian TE, Ferri GL, Bacarese-Hamilton AJ, et al. (1985) Human distribution and release of a putative new gut hormone, peptide YY. Gastroenterology 89, 1070-1077.

44. Saad MF, Riad-Gabriel MG, Khan A, et al. (1998) Diurnal and ultradian rhythmicity of plasma leptin: effects of gender and adiposity. J Clin Endocrinol Metab 83, 453-459.

45. Schoeller DA, Cella LK, Sinha MK, et al. (1997) Entrainment of the diurnal rhythm of plasma leptin to meal timing. J Clin Invest 100, 1882-1887.

46. Blom WA, Stafleu A, de Graaf C, et al. (2005) Ghrelin response to carbohydrate-enriched breakfast is related to insulin. Am J Clin Nutr 81, 367-375.

47. Foster-Schubert KE, Overduin J, Prudom CE, et al. (2008) Acyl and total ghrelin are suppressed strongly by ingested proteins, weakly by lipids, and biphasically by carbohydrates. J Clin Endocrinol Metab 93, 1971-1979.

48. Flanagan DE, Evans ML, Monsod TP, et al. (2003) The influence of insulin on circulating ghrelin. Am J Physiol Endocrinol Metab 284, E313-E316.

49. Murdolo G, Lucidi P, et al. (2003) Di Loreto C. Insulin is required for prandial ghrelin suppression in humans. Diabetes 52, 2923-2927.

50. Saad MF, Bernaba B, Hwu CM, et al. (2002) Insulin regulates plasma ghrelin concentration. J Clin Endocrinol Metab 87, 3997-4000

51. Caixas A, Bashore C, Nash W, et al. (2002) Insulin, unlike food intake, does not suppress ghrelin in human subjects. J Clin Endocrinol Metab 87, 1902.
52. Schaller G, Schmidt A, Pleiner J, et al. (2003) Plasma ghrelin concentrations are not regulated by glucose or insulin: a double-blind, placebo-controlled crossover clamp study. Diabetes 52, 16-20.

53. Blom WA, de Graaf C, Lluch A, et al. (2009) Postprandial ghrelin responses are associated with the intermeal interval in time-blinded normal weight men, but not in obese men. Physiol Behav 96, 742-748.

54. Hamman L \& Hirschmann I (1919) Studies on blood sugar. IV. Effects upon the blood sugar of the repeated ingestion of glucose. Johns Hopkins Hosp Bull 30, 306-307.

55. Bonuccelli S, Muscelli E, Gastaldelli A, et al. (2009) Improved tolerance to sequential glucose loading (Staub-Traugott effect): size and mechanisms. Am I Physiol Endocrinol Metab 297, E532-E537.

56. Reeves S, Huber JW, Halsey LG, et al. (2014) Experimental manipulation of breakfast in normal and overweight/obese participants is associated with changes to nutrient and energy intake consumption patterns. Physiol Behav 133C $130-135$.

57. Robertson MD, Henderson RA, Vist GE, et al. (2002) Extended effects of evening meal carbohydrate-to-fat ratio on fasting and postprandial substrate metabolism. Am J Clin Nutr 75, 505-510.

58. Hochstenbach-Waelen A, Veldhorst MA, Nieuwenhuizen AG, et al. (2009) Comparison of 2 diets with either $25 \%$ or $10 \%$ of energy as casein on energy expenditure, substrate balance, and appetite profile. Am J Clin Nutr $\mathbf{8 9}$, $831-838$. 